Editorial

\title{
Why status of stunting is not improving in Bangladesh
}

\section{Editorial}

Stunting is defined as low height for age. Stunting is a cumulative effect of undernutrition and infections before and after birth. Along with long-term undernutrition, this measure is an indication of poor environmental conditions that encourages repeated infections. Stunting is considered as serious public health problem in Bangladesh. Stunting results decreased learning capacity as well as earning capacity which ultimately decreases national productivity. According to Bangladesh Demographic and Health survey prevalence of stunting is still high in the country. Once upon a time it is thought that undernutrition is responsible for stunting but research suggests that undernutrition contribute only $30 \%$ and rest $70 \%$ is environmental enteropathy which reduce capacity for nutrient absorption may lead to stunting. We always emphasize on diet and nutrition of child but neglect environmental factors. Actually stunting never occurs suddenly rather it is a chronic condition and intestinal infection play major role to develop stunting. Studies show that women who had been stunted give birth to babies with low birth weight. There is also evidence that babies born to stunted women are at a greater risk of dying than children born to women of normal height. In field level we observe that emphasize is given on pregnant mother and child but not on lactating mother results low breast milk production followed by inadequate breast feeding upto six months and early initiation of complementary feeding. It is fact that lactating mother needs more energy than pregnant women. Still there is misconception among
Volume 4 Issue 5 - 2016

Sanjeev Kumar Shah

Senior Lecturer, Community Medical Institute, Bangladesh

Correspondence: Md Monoarul Haque, Senior Lecturer Community Medical Institute, Bangladesh, Tel 8801915839550; Email monoarmunna@yahoo.com

Received: June 21, 2016 | Published: June 23, 2016

mothers about exclusive breast feeding and they think that only breast milk is not enough for baby; peer group pressure is another contributing factor. So if we want to improve stunting status in our country we have to go for holistic approach like diet, WASH and environment-all together.

\section{Acknowledgements}

None.

\section{Conflict of interest}

The author declares no conflict of interest. 